\title{
Chapter 13 \\ Hydrogel-Based Biomimetic Environment for In Vitro Cell and Tissue Manipulation
}

\author{
Takuya Matsumoto
}

\begin{abstract}
A biomimetic environment fabricated with synthetic material would be an effective tool for reproducing the tissue-developmental process and even for achieving tissue engineering in vitro. A hydrogel material is one candidate for this tool, because a hydrogel normally shows harmless properties in regard to cells and tissue, and it can be tuned chemically and physically to obtain the desired form. Accordingly, fibrin gel was utilized to reproduce the 3D cellular orientations found in muscle tissue, fabricate tendon-like mineralized tissue, and regulate vascular formation. In this context, cell and tissue manipulations within the gel were led by in vitro physical and chemical stimulations. In this chapter, the approach used for manipulating cells and tissues using the designed hydrogel is discussed.
\end{abstract}

Keywords Biomimetic environment - Cell manipulation - Hydrogel • In vitro tissue engineering

\subsection{Introduction}

Thanks to recent advances in cell and molecular biology, researchers have gradually started to understand how molecules are concerned with the expression of cellular functions. They have also started to understand how the surrounding molecules guide cellular behavior. Engineers and chemists have also started to participate in this in-vitro cellular guidance, develop methods for so-called "in-vitro cellular guidance," since they can design and construct an environment that is suitable for manipulating cell functions. For example, Chen et al. used a microprinting system that can control the cell-adhesion shape by applying the patterned coating of fibronectin on two-dimensional tissue-culture substrates. They indicated that the mesenchymal stem cells (MSCs) shape regulates the switch in lineage commitment by modulating endogenous RhoA activity. Expressing dominant-negative RhoA committed MSCs to become adipocytes, while constitutively active RhoA caused

\footnotetext{
T. Matsumoto $(\triangle)$

Department of Biomaterials, Okayama University, 2-5-1 Shikata-cho, Kita-ku,

Okayama 700-8558, Japan

e-mail: tmatsu@md.okayama-u.ac.jp
} 
osteogenesis [1]. Discher and Mooney indicated that hydrogel with different mechanical stiffnesses regulate cell proliferation, cell differentiation, and even the uptakes of non-viral vectors [2-4]. Since the environment surrounding a cell can be easily tuned by materials and devices according to our favorable design, a newly developed research methodology (integrative biology) is now recognized as a newcomer to find something new that cannot be found by conventional biological methods $[5,6]$. Here, we consider that reproducing the tissue-development process in vitro is investigated as a robust tool for understanding cellular behavior in the tissue-developmental stage so that in vitro tissue engineering becomes possible.

\subsection{Cell and Matrix Patterning Using Hydrogel with Static Mechanical Stimulation}

Specific cellular and matrix patterning can be found in biological tissue development and growth. For example, skeletal muscle tissue consists of cells aligned parallel to the long axis of the muscle tissue. Osteoblasts adhered to the surface of bone matrix align in a similar direction to the bone-tissue growth direction in longitudinal bone. Reproduction of parallel cell alignment in three-dimensional (3D) matrices is thus one of the interesting targets for in-vitro tissue engineering. Hydrogel contains more than $80 \%$ water, which is crucial for exchange of nutrition between cells and has similar mechanical properties to those of biological tissue. Fibrin, which is formed by mixing fibrinogen and thrombin purified from peripheral blood, is found in wound-healing regions. Not only is it used therapeutically as surgical glue but fibrin has also been investigated for its use as a biocompatible and biodegradable material in biomedical-engineering applications (e.g., drug delivery systems [7-9] and tissue engineering [10-12]). Fibrin gels, which are comprised of hydrophilic cross-linked fibrils, are considered suitable for 3D cell culture. To obtain 3D matrices having uniformly aligned fibrin fibers, surgical sutures fabricated from poly (lactic-co-glycolic acid) (PLGA) were used to tether the fibrin gels. Each fibrin gel was formed in a cylindrical silicone mold (length: $10 \mathrm{~mm}$, diameter: $6 \mathrm{~mm}$ ) with sutures inserted at both ends. The sutures were then clamped to a custom-made device for generating continuous tensile strain (up to $200 \%$ ) (Fig. 13.1a). The suture material possessed a highly rough texture due to its woven structure, and it firmly attached to the fibrin matrices. Scanning electron microscopy (SEM) images indicated that bundle-like structures were formed in the strained gels; this structure comprised fibrin fibrils that were oriented parallel to the strain direction (Fig. 13.1b, c). Highly magnified SEM images indicated that the fibril alignment in the bundle-like structure depend on the amount of strain applied. The fibrils were torn at the border of each bundle, suggesting that the displacement of each set of fibrils in the strain direction facilitated the formation of bundle-like structures in the fibrin gel. The cross-section of the generated bundle-like structures exhibited a polygonal shape, not a complete circular shape, which is similar to the cross-section of natural skeletal muscle tissue. The estimated diameter of each bundle-like structure decreased with increasing strain [13]. 

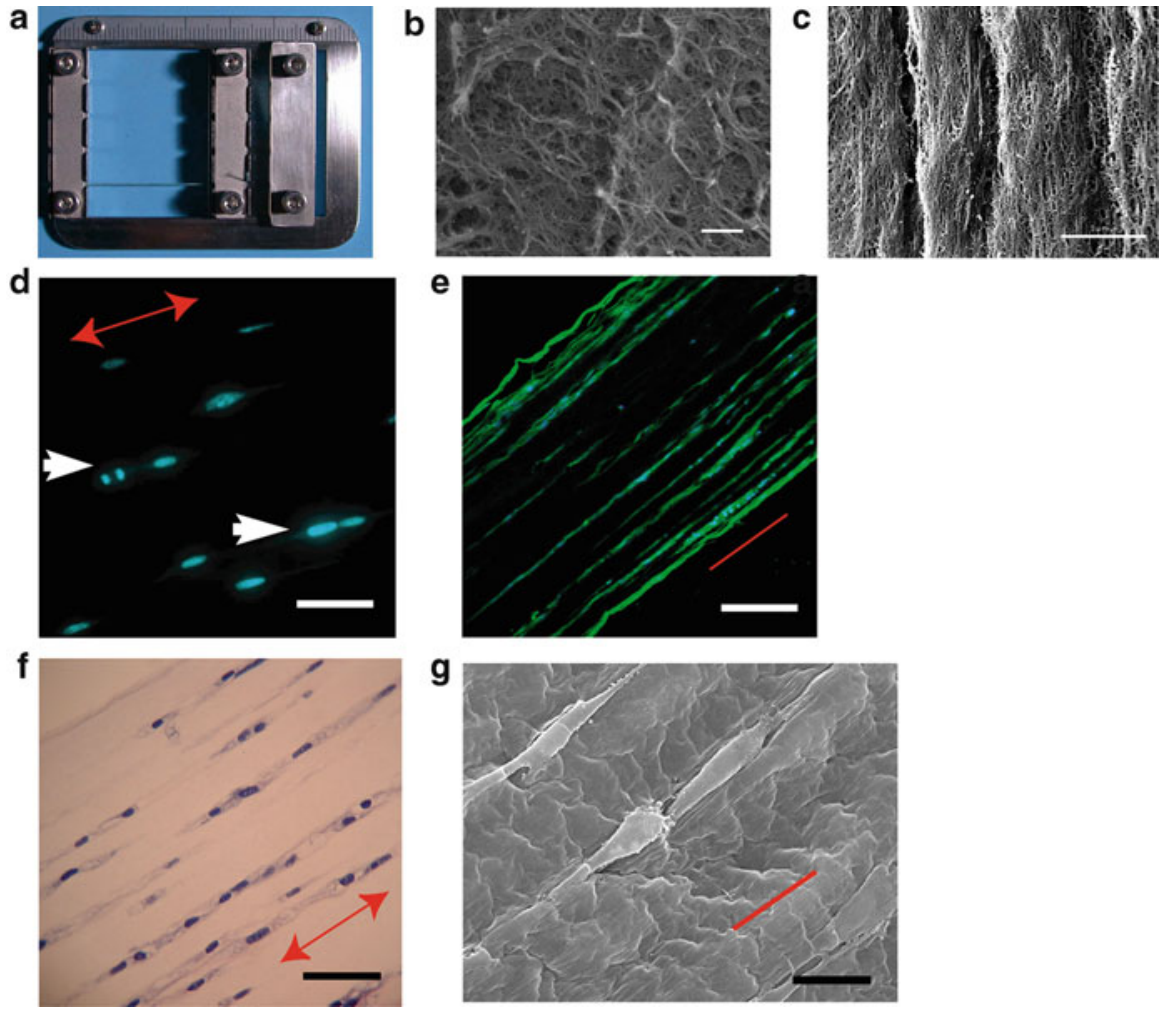

Fig. 13.1 (a) Custom made device fabricated for tethering the strained fibrin gel. (b) The representative SEM image of microstructure of fibrin gel without strain (Bar: $1 \mu \mathrm{m}$ ). (c) The representative SEM image of strained fibrin gel (Bar: $5 \mu \mathrm{m})$. (d) The direction of cell proliferation was also identical with strain direction of hydrogel (Bar: $100 \mu \mathrm{m})$. (e) Threedimensionally aligned cell groups formed in the strained fibrin gel (Green; actin, Blue; nucleus) (Bar: $400 \mu \mathrm{m}$ ). (f) Three- dimensionally aligned cell groups formed in the strained fibrin gel (Hematoxylin-Eosin staining) (Bar: $200 \mu \mathrm{m})$. (g) SEM images of aligned cells in the strained fibrin gel (Bar: $100 \mu \mathrm{m})$

\subsection{Three-Dimensional Patterning of Mineralized Cell Groups in Hydrogel}

The cell dynamics within the strained fibrin gel were investigated. The fibrinogen solution containing myoblast $(\mathrm{C} 2 \mathrm{C} 12)$ was used to form a gel, which was continuously subjected to $25 \%$ strain. The cells in the fibrin gel display a specific alignment, that is, parallel to the strain direction. Interestingly, the direction of cell proliferation was identical to that of cell alignment (Fig. 13.1d). A single seeded cell therefore divided multiple times, and the oriented cells subsequently formed linear groups aligned parallel to the strain direction (Fig. 13.1e, f), in a similar manner to the cellular organization found in a longitudinal section of native 
skeletal muscle tissue. It is assumed that the positions of the cells in the fibrin gel are restricted to the spaces between the fibrin bundles such that they align and proliferate parallel to the strain direction. This assumption is supported by a typical SEM image (Fig. 13.1g), which shows cells positioned in the spaces between the bundle-like structures of the fibrin gel.

In light of the above-described results, bone-marrow stromal cells (BMSCs) we used instead of myoblasts to fabricate the aligned mineralized tissue. In a similar manner to the cells in the above-described myoblast study, the cells in the strained gel displayed a specific alignment, namely, parallel to the strain direction in the gel. The direction of cell proliferation was identical to that of cell alignment. Consequently, the oriented cells formed a number of linear cell groups aligned parallel to the strain direction in the strained gel. In the strained fibrin gel, type-I collagen deposition showed a specific orientation, namely, parallel to the strain direction (Fig. 13.2a, b). The merged images with nuclear-stained cells in the figure indicate that the matrix was deposited in identical positions to the cell positions in the gel. When BMSCs were cultured in an osteogenic differentiation medium, mineralization derived from the cells was observed in the fibrin gel. Similar to the matrix deposition, mineral deposition was localized according to the cell position and showed a specific alignment in the strained gel. X-ray-diffraction (XRD) peak profiles revealed that the obtained mineral in the fibrin gel was hydroxyapatite (HAp) (Fig. 13.2c). Additionally, a specific orientation of the HAp crystals (namely, parallel to the strain direction) was confirmed from the relative intensity of the (002) to (211) planes in the XRD profiles (Fig. 13.2c). SEM images indicate that compared to those in the control gel, the mineralized matrix vesicles were concatenated serially parallel to the strain direction in the strained gel (Fig. 13.2d). EDS analysis revealed that the mineralized matrix vesicles contain both calcium and phosphorus. The concentrations of phosphorus in the mineralized vesicle regions are much higher than those of calcium in that region.

To investigate the alternations of cellular functions in strained fibrin gel, the cells were cultured in gels with different strain rates. At day eight, the gel subjected to a higher strain rate had enhanced cell proliferation compared to the gel subjected to a lower strain rate. The mRNA expressions of Opn and Oc, namely, osteogenic differentiation markers, were investigated at day four. Both Opn and Oc expressions decreased with the increasing strain rate from up to $50 \%$. These results suggest that cell functions in the strained fibrin gel are regulated by the alteration of strain rate. To confirm this suggestion, cell-derived mineralization in the gel (subjected to varied strain rate during the culture period) was investigated. Mineralization caused by cell differentiation was detected only in the sample that was subjected to strain rate decreased from 50 to $0 \%$ at day 21 (III). In contrast, mineral deposition was not detected in the gel that was subjected to strain rate reduced from 50 to $0 \%$ at day 28 (II) or in the gel that was subjected to $50 \%$ strain maintained for 50 days (I) (Fig. 13.2e) [14]. 
a

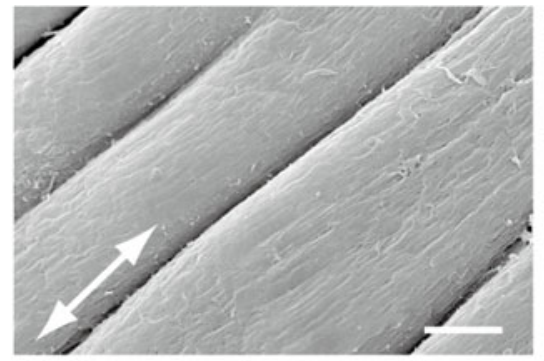

C

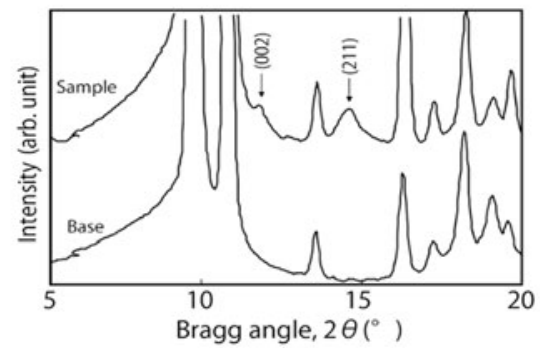

b

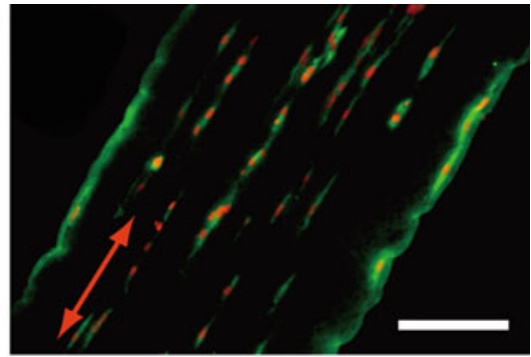

d

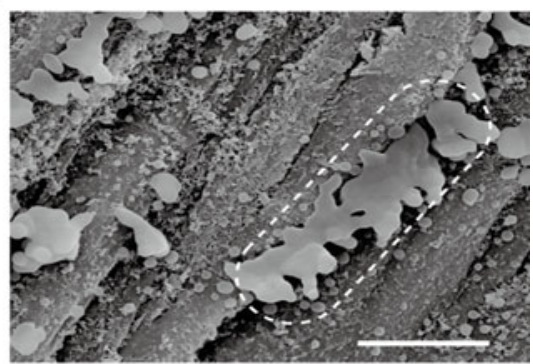

Mineralized area (\%)

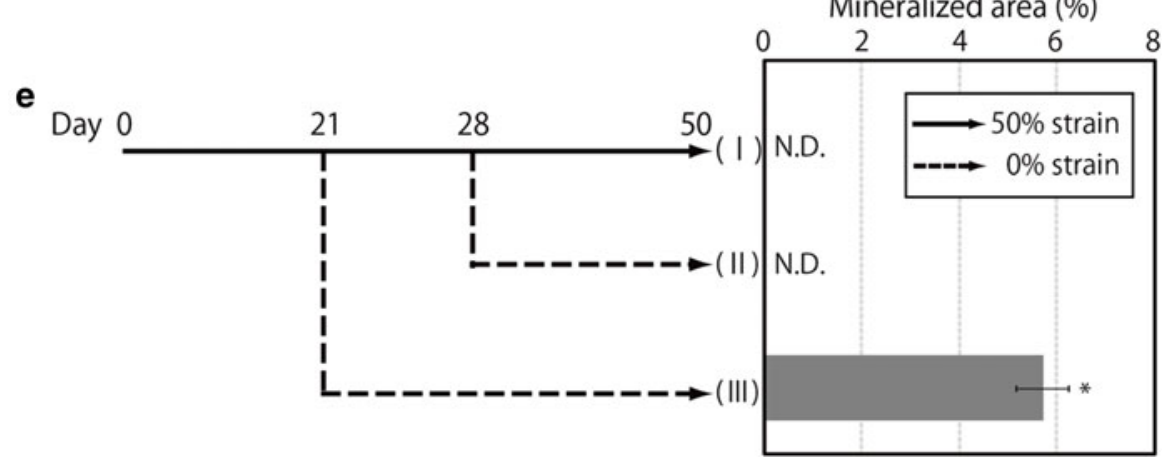

Fig. 13.2 (a) SEM images of bundle-like structure formed in the strained fibrin gel (Bar: $20 \mu \mathrm{m}$ ). (b) Aligned cell groups and precipitated type I collagen according to the cell group presence (Bar: $250 \mu \mathrm{m})$. (c) X-ray diffraction analysis of precipitated minerals within the strained fibrin gel. (d) Connected minerals on aligned cells and fibers (Bar: $10 \mu \mathrm{m})$. (e) Mineralized area in strained fibrin gel cultured with different strain conditions. (I) $50 \%$ strain for 50 days, (II) $50 \%$ strain for 28 days reduced to $0 \%$ strain for another 22 days, and (III) $50 \%$ strain for 21 days reduced to $0 \%$ strain for another 29 days $(*: \mathrm{p}<0.05)$

\subsection{Microvessel Patterning Using Fibrin Gel with Dynamic Mechanical Stimulation}

By here, the cells were cultured in the strained condition; however, the strain was static and continuous. A device for applying dynamic strain against to cells in 3D hydrogel was also designed. The device was used to investigate the formation of 

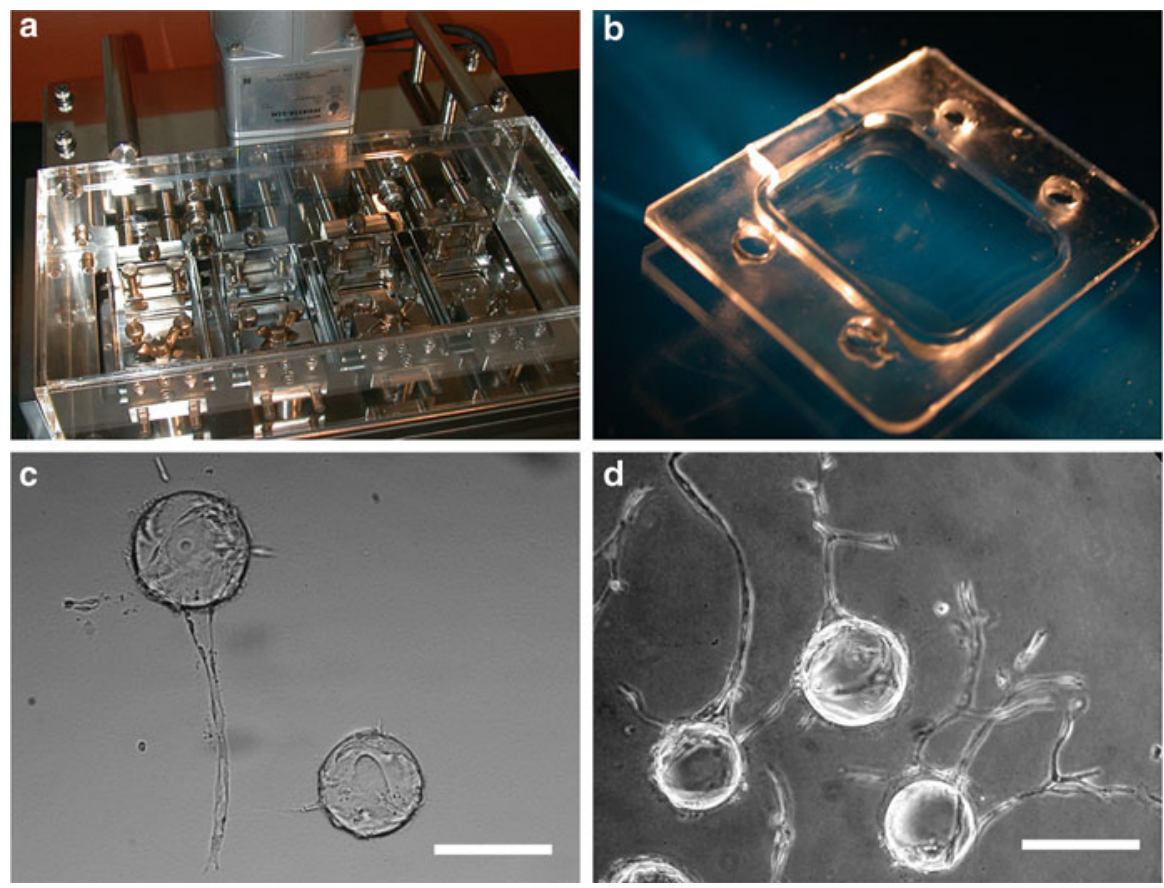

Fig. 13.3 (a) Original device for applying mechanical strain to cells in the 3D gel. (b) Chamber for cell culture in 3D gel with strained condition. (c) Newly formed sprouts with well defined lumens elongated perpendicular to the strain direction when subjected to cyclic strain (Bar: $100 \mu \mathrm{m})$. (d) Cells in static culture sprouted in all directions equally (Bar: $100 \mu \mathrm{m}$ )

vascular tissue under cyclic mechanical stimulations. Dextran beads coated with human umbilical endothelial cells (HUVECs) were embedded in fibrin gels within a custom 3D chamber and subjected to cyclic strain (Fig. 13.3a, b). HUVECs on the beads started to invade the gels in a direction perpendicular to the strain direction and formed sprout-like structures within 5 days (Fig. 13.3c). Each bead formed only one or two of these structures, and they were aligned in a direction predominantly perpendicular to the direction of strain application. There were no branches in these structures formed in the strained condition. In contrast, single cells migrated, proliferated, and formed multiple sprouts per bead in the static condition. These sprouts had no consistent orientation, and the sprouts formed in this condition were branched (Fig. 13.3d). Structures formed in the presence of cyclic strain were also significantly thicker than those formed in the static condition. The structures formed under cyclic strain contained wall cells with nuclei located toward the some alternatives lumens, but the lumens also contain aligned cells. These cells were likely proliferated inside the structures, while the wall cells migrated and proliferated to form the structures [15]. 


\subsection{Conclusion}

As mentioned here, the hydrogel system can be physically tuned by applying mechanics. The cultured cells used in the physical stimulations show different behavior according to the surrounding architectures or stimulation conditions. Conventionally, the cell behavior was regulated only to confirm the effect of soluble factors that were newly-cloned. However, recent studies aiming to modulate cells and tissue to fabricate cell-based functional materials, or even to achieve biological tissue synthesis in vitro, have been performed [16-21]. In the present study, as well as chemical stimulation, physical stimulation is also considered as a promising candidate to modulate cell and tissue functions. Moreover, the trials on these cell and tissue manipulations would be valuable to help understanding of the biological unknown during the tissue-developmental process.

Open Access This chapter is distributed under the terms of the Creative Commons Attribution Noncommercial License, which permits any noncommercial use, distribution, and reproduction in any medium, provided the original author(s) and source are credited.

\section{References}

1. McBeath R, Pirone DM, Nelson CM, Bhadriraju K, Chen CS. Cell shape, cytoskeletal tension, and RhoA regulate stem cell lineage commitment. Dev Cell. 2004;6:483-95.

2. Engler AJ, Sen S, et al. Cell. 2006;126:677-89.

3. Kong HJ, Polte TR, Alsberg E, Mooney DJ. FRET measurements of cell-traction forces and nano-scale clustering of adhesion ligands varied by substrate stiffness. Proc Natl Acad Sci U S A. 2005;102:4300-5.

4. Kong HJ, Matsumoto T, et al. Nat Mater. 2005;4:460-4.

5. Lutolf MP. Integration column: artificial ECM: expanding the cell biology toolbox in 3D. Integr Biol (Camb). 2009;1:235-41.

6. Beebe D, Cheran L. Introducing a new method section for integrative biology. Integr Biol (Camb). 2009;1:445.

7. Lu X, Le Noble F, Yuan L, Jiang Q, De Lafarge B, Sugiyama D, et al. The netrin receptor UNC5B mediates guidance events controlling morphogenesis of the vascular system. Nature. 2004;432:179-86.

8. Weinstein BM. Vessels and nerves: marching to the same tune. Cell. 2005;120:299-302.

9. Alon T, Hemo I, Itin A, Pe'er J, Stone J, Keshet E. Vascular endothelial growth factor acts as a survival factor for newly formed retinal vessels and has implications for retinopathy of prematurity. Nat Med. 1995;1:1024-8.

10. Senger DR, Ledbetter SR, Claffey KP, Papadopoulos-Sergiou A, Peruzzi CA, Detmar M. Stimulation of endothelial cell migration by vascular permeability factor/vascular endothelial growth factor through cooperative mechanisms involving the alphavbeta3 integrin, osteopontin, and thrombin. Am J Pathol. 1996;149:293-305.

11. Grunstein J, Masbad JJ, Hickey R, Giordano F, Johnson RS. Isoforms of vascular endothelial growth factor act in a coordinate fashion to recruit and expand tumor vasculature. Mol Cell Biol. 2000;20:7282-91.

12. Gerhardt H, Golding M, Fruttiger M, Ruhrberg C, Lundkvist A, et al. VEGF guides angiogenic sprouting utilizing endothelial tip cell filopodia. J Cell Biol. 2003;161:1163-77. 
13. Matsumoto T, Sasaki J, Alsberg E, Egusa H, Yatani H, Sohmura T. Three-dimensional cell and tissue patterning in a strained fibrin gel system. PLoS One. 2007;2:e1211.

14. Sasaki JI, Matsumoto T, Egusa H, Nakano T, Ishimoto T, Sohmura T, et al. In vitro engineering of transitional tissue by patterning and functional control of cells in fibrin gel. Soft Matter. 2010;6:1662-7.

15. Matsumoto T, Yung YC, Fischbach C, Kong HJ, Nakaoka R, Mooney DJ. Mechanical strain regulates endothelial cell patterning in vitro. Tissue Eng. 2007;13:207-17.

16. Matsumoto T, Mooney DJ. Cell instructive polymers. In: Kaplan D, editor. Tissue engineering, Advances in biochemical engineering/biotechnology series, vol. 102. Berlin: Springer; 2006. p. 113-37.

17. Eiraku M, Takata N, Ishibashi H, Kawada M, Sakakura E, Okuda S, et al. Self-organizing optic-cup morphogenesis in three-dimensional culture. Nature. 2011;472:51-6.

18. Sasaki J, Matsumoto T, Egusa H, Matsusaki M, Nishiguchi A, Nakano T, et al. In vitro reproduction of endochondral ossification using a 3D mesenchymal stem cell construct. Integr Biol (Camb). 2012;4:1207-14.

19. Miyajima H, Matsumoto T, Sakai T, Yamaguchi S, An SH, Abe M, et al. Hydrogel-based biomimetic environment for in vitro modulation of branching morphogenesis. Biomaterials. 2011;32:6754-63.

20. Higgins CA, Chen JC, Cerise JE, Jahoda CA, Christiano AM. Microenvironmental reprogramming by three-dimensional culture enables dermal papilla cells to induce de novo human hair-follicle growth. Proc Natl Acad Sci U S A. 2013;110:19679-88.

21. Ogawa M, Oshima M, Imamura A, Sekine Y, Ishida K, Yamashita K, et al. Functional salivary gland regeneration by transplantation of a bioengineered organ germ. Nat Commun. 2013;4:2498. 